\title{
Effective Multimodal Feedback for Around-Device Interfaces
}

\author{
Euan Freeman \\ Glasgow Interactive Systems Group \\ University of Glasgow \\ Glasgow, Scotland \\ euan.freeman@glasgow.ac.uk
}

\begin{abstract}
Around-device interaction lets people use their phones without having to pick them up or reach out and touch them. This allows interaction when touch may not be available; for example, users could gesture to interact with their phones while cooking, avoiding touching the screen with messy hands. Well-designed feedback helps users overcome uncertainty during gesture interaction, however giving effective feedback from small devices can be a challenge and detailed visual feedback will not always be suitable. My thesis research looks at how other types of feedback may be used effectively during around-device interaction to help users.
\end{abstract}

\section{Author Keywords}

Around-device interaction; multimodal; tactile feedback.

\section{ACM Classification Keywords}

H.5.2. User Interfaces: Interaction styles.

\section{INTRODUCTION}

Gesture interfaces are being introduced to mobile phones to allow more expressive input and to let users interact when using the touchscreen is impractical. Researchers have developed a variety of sensing techniques for around-device interaction and recently Samsung included a gesture interface in their Galaxy S4 phone. As sensors continue to improve, especially in terms of power requirements, more mobile phones are likely to let users interact this way.

Providing effective feedback is important to help users overcome uncertainty during gesture interaction; however, giving effective feedback from such a small device can be challenging. Small screens limit visual feedback and mean feedback may not even be visible if users are occluding the display with their hand or gesturing from a short distance.

My research looks at how other output types can be used to give effective feedback during gesture interaction, to complement visual feedback and to help users when visual feedback is unavailable. While others have used non-visual feedback in around-device interfaces before (e.g. tactile [6] and

This is the author's copy. The definitive version of this work can be found in the MobileHCI 2014 proceedings in the ACM Digital Library.

MobileHCI 2014, September 23-26, 2014, Toronto, ON, Canada. ACM 978-1-4503-3004-6/14/09.

http://dx.doi.org/10.1145/2628363.2634264 speech [3]), research is needed to explore feedback design in greater detail and to understand what information users actually need to help them gesture and support their awareness of how the interface is responding to their actions.

Here I will discuss my thesis research, which looks at how other feedback types can be used effectively in around-device interfaces. I will outline my research goals, discuss my progress towards these goals from the first 18 months of my $\mathrm{PhD}$, and talk about my plan for the remaining 18 months.

\section{RESEARCH GOALS AND APPROACH}

Multimodal feedback can address limitations with visual feedback, helping users overcome uncertainty and gesture more effectively. My thesis research aims to learn how other modalities can be used effectively in around-device interfaces. I will look at three output types in particular: tactile, audio and ambient visual. Tactile and audio feedback are commonly used in other interaction techniques, although tactile in particular has its own challenges when given in a gesture interface. Ambient visual feedback uses embedded lights (as in [7]) to illuminate space around a phone, creating visual cues which are noticeable from a distance and do not take space away from on-screen content.

I will look at what types of information users need during gesture interaction and how each modality can be used to deliver this. Each feedback type will have its own strengths and weaknesses in this context so multiple outputs may be needed to support users. I will conduct my research by creating multimodal around-device interfaces and then evaluating them in order to understand how effective feedback is. A mixture of quantitative and qualitative methods will give insight into what users like and dislike about each type of output, and what information they need to support them while gesturing. This mixed-methods approach is appropriate for this research area because user experience is important but difficult to understand through quantitative methods alone. User experience can also motivate feedback design because users may find certain feedback helpful even though it results in no performance improvement, for example.

\section{RESEARCH STATUS}

\section{Completed Work}

First I looked at designing usable and acceptable gestures for mobile phones [2]. Then I completed two studies, both focusing on tactile feedback for around-device interfaces. These studies looked at tactile feedback for selection, as this is a common around-device interaction (e.g. $[1,4,3])$. 
My first study looked at two ways of giving tactile feedback in a gesture interface: non-contact feedback using ultrasound haptics [5] and distal feedback from wearables. As well as looking at delivery, this study also compared two feedback designs. This study showed that all feedback types had similar workload (NASA-TLX) and performance (selection time and error rate), with only ultrasound feedback having significantly worse performance. Participants found tactile feedback useful because it showed system attention and supported their awareness of how the interface was responding to their gestures. Preference for feedback method and designs varied.

My second study looked at refined feedback designs, inspired by participants' suggestions from the first study. Three feedback designs were created: two which encoded selection progress tactually (using amplitude and vibrotactile roughness) and one which presented constant stimulus. The constant feedback design represented the type of tactile feedback currently available from wearables, where vibration can only simply be turned on or off. Feedback had little effect on performance but did have a significant effect on workload: dynamic feedback (those encoding selection progress) had lower workload than when no tactile feedback was given. There were no significant differences between constant feedback and no feedback, and between constant feedback and dynamic feedback. Participants also ranked all feedback types significantly higher than no tactile feedback. As before, participants said tactile feedback supported their awareness of how the interface was affected by their movements and they found rich feedback designs helpful.

These studies were a first detailed look at tactile feedback in around-device interfaces. Tactile feedback generally improves the user experience of gesturing and can lower the workload of around-device interaction. Wearables show promise as tactile displays because they can be worn in a variety of form factors and my findings show that feedback at different locations (on the wrist and on the finger) can be effective. My second study looked at refined feedback designs and found that feedback encoding selection progress reduced the workload of around-device selection. As wearable technologies grow, wearable developers should consider letting designers control more vibrotactile parameters than simply turning vibration on and off, allowing these devices to be used as rich tactile displays for other interaction modalities.

\section{Remaining Work}

In the remaining 18 months of my $\mathrm{PhD}$ I will look at audio and ambient visual feedback for around-device interfaces. I am currently running a study which takes a first look at ambient visual feedback in gesture interfaces. This study will look at how this new output type can be used to give feedback during gesture input in smart-home devices. Preliminary results suggest that users find ambient visual feedback helpful, especially when complemented by audio or tactile feedback. Further studies will take lessons learned and apply them to mobile phones, further exploring the design space and effectiveness of ambient visual feedback.

Further studies will also look at audio feedback design. These will focus on designing useful feedback as well as looking at the acceptability of audio feedback. Continuous audio feedback, while rich in information, could be obtrusive and annoying to nearby people so must convey useful information in an acceptable way. Finally, I will take lessons learned about each modality and create a multimodal interface which brings together these three modalities to give effective feedback.

\section{CONCLUSION}

My thesis research looks at what feedback is needed to help users when gesturing with mobile phones and how this feedback may be given. So far I have looked at how tactile feedback may be designed and presented in around-device interfaces. In the remaining eighteen months of my PhD I plan to focus more on ambient visual feedback and audio feedback, to see how these can be used to support interaction.

Attending the DC would let me engage with and get valuable feedback from other students and leading researchers in the field. As I am approaching the middle of my three-year PhD this would be an ideal time to get feedback, to help me make a stronger contribution from my research. I would particularly like to discuss how to frame my findings as the main contribution of my thesis: would a series of design recommendations be more or less useful than a taxonomy of the design space which lets others decide how to approach and extend the design of around-device feedback?

\section{ACKNOWLEDGEMENTS}

This research is funded by Nokia Research Center, Finland. Many thanks to Vuokko Lantz and Stephen Brewster for their supervision and support.

\section{REFERENCES}

1. Butler, A., Izadi, S., and Hodges, S. SideSight: Multi-touch Interaction Around Small Devices. In Proc. UIST '08, ACM Press (2008), 201-204.

2. Freeman, E., Brewster, S., and Lantz, V. Towards Usable and Acceptable Above-Device Interactions. In Proc. Mobile HCI '14 Posters, ACM Press (2014), to appear.

3. Gustafson, S., Holz, C., and Baudish, P. Imaginary Phone: Learning Imaginary Interfaces by Transferring Spatial Memory from a Familiar Device. In Proc. UIST'11, ACM Press (2011), 283-292.

4. Harrison, C., and Hudson, S. E. Abracadabra: Wireless, High-Precision, and Unpowered Finger Input for Very Small Mobile Devices. In Proc. UIST '09, ACM Press (2009), 121-124.

5. Hoshi, T., Takahashi, M., Iwamoto, T., and Shinoda, H. Noncontact Tactile Display Based on Radiation Pressure of Airborne Ultrasound. IEEE Transactions on Haptics 3, 3 (2010), 155-165.

6. Niikura, T., Watanabe, Y., Komuro, T., and Ishikawa, M. In-air Typing Interface: Realizing 3D operation for mobile devices. In Proc. GCCE '12, IEEE (2012), 223-227.

7. Qin, Q., Rohs, M., and Kratz, S. Dynamic Ambient Lighting for Mobile Devices. In Adjunct Proc. UIST'11, ACM Press (2011), 51-52. 